\title{
Instant Preheating
}

\author{
Gary Felder, ${ }^{1}$ Lev Kofman, ${ }^{2,3}$ and Andrei Linde ${ }^{1}$ \\ ${ }^{1}$ Department of Physics, Stanford University, Stanford, CA 94305-4060, USA \\ ${ }^{2}$ CITA, University of Toronto, 60 St George Str, Toronto, ON M5S 1A1, Canada \\ ${ }^{3}$ CEA/Saclay, Service de Physique Théorique, F-91191 France
}

(December 8, 1998)

\begin{abstract}
We describe a new efficient mechanism of reheating. Immediately after rolling down the rapidly moving inflaton field $\phi$ produces particles $\chi$, which may be either bosons or fermions. This is a nonperturbative process which occurs almost instantly; no oscillations or parametric resonance is required. The effective masses of the $\chi$ particles may be very small at the moment when they are produced, but they "fatten" when the field $\phi$ increases. When the particles $\chi$ become sufficiently heavy, they rapidly decay to other, lighter particles. This leads to an almost instantaneous reheating accompanied by the production of particles with masses which may be as large as $10^{17}-10^{18} \mathrm{GeV}$. This mechanism works in the usual inflationary models where $V(\phi)$ has a minimum, where it takes only a half of a single oscillation of the inflaton field $\phi$, but it is especially efficient in models with effective potentials slowly decreasing at large $\phi$ as in the theory of quintessence.
\end{abstract}

PACS: $98.80 . \mathrm{Cq} \quad$ SU-ITP-98-65 hep-ph/9812289

\section{INTRODUCTION}

During the last few years we have learned that the first stages of reheating after inflation are typically governed by nonperturbative effects. In particular, the most efficient mechanism of reheating which was known until now was based on the theory of the nonperturbative decay of the inflaton field due to the effect of broad parametric resonance [1]. To distinguish this stage of nonperturbative particle production from the stage of particle decay and thermalization which can be described using perturbation theory [2] (see also [3]), it was called preheating.

This process can rapidly transfer the energy of a coherently oscillating scalar field to the energy of other fields or elementary particles. Because of the nonperturbative nature of the process, it may lead to many unusual effects, such as nonthermal cosmological phase transitions [4. Another unusual feature of preheating discovered in [1] is the possibility of the production of a large amount of superheavy particles with masses one or two orders of magnitude greater than the inflaton mass. In the simplest versions of chaotic inflation with the inflaton mass $m \sim 10^{13} \mathrm{GeV}$ this can lead to the copious production of particles with masses up to $10^{14}-10^{15} \mathrm{GeV}$ [1, 5] . This issue is rather important since interactions and decay of superheavy particles may lead to baryogenesis at the GUT scale [5].

However, GUT baryogenesis was only marginally possible in the models of preheating studied until now because the masses of produced particles just barely approached the GUT scale. Moreover, in some models the particles created by the resonance strongly interact with each other, or rapidly decay. This may take them out of the resonance band, in which case the parametric resonance does not last long or does not happen at all. Also, there are some models where the effective potential does not have a minimum, but instead slowly decreases at large $\phi$ [8,9]. In these models the scalar field does not oscillate at all after inflation, so neither parametric resonance nor the standard perturbative mechanism of inflaton decay works there.

In this paper we will try to turn these potential problems into an advantage. We will describe a new mechanism of preheating, which works even in the models where parametric resonance cannot develop. The new mechanism is also nonperturbative but very simple. It leads to an almost instantaneous reheating accompanied by the production of superheavy particles with masses which may be as great as $10^{17}-10^{18} \mathrm{GeV}$. In some cases it may even lead to the production of black holes of a Planckian mass, which immediately evaporate.

\section{INSTANT PREHEATING: THE BASIC IDEA}

To explain the main idea of the new scenario we will consider the simplest model of chaotic inflation with the effective potential $\frac{m^{2}}{2} \phi^{2}$ or $\frac{\lambda}{4} \phi^{4}$ and assume that the inflaton field $\phi$ interacts with some other scalar field $\chi$ with the interaction term $-\frac{1}{2} g^{2} \phi^{2} \chi^{2}$. In these models inflation occurs at $|\phi| \gtrsim 0.3 M_{p}[10]$. Suppose for definiteness that initially $\phi$ is large and negative, and inflation ends at $\phi \sim-0.3 M_{p}$. After that the field $\phi$ rolls to $\phi=0$, then it grows up to $10^{-1} M_{p} \sim 10^{18} \mathrm{GeV}$, and finally rolls back and oscillates about $\phi=0$ with a gradually decreasing amplitude. If the coupling constant $g$ is large enough $\left(g \gtrsim 10^{-4}\right)$, then, according to [1], the production of particles $\chi$ occurs for the first time when the scalar field $\phi$ reaches the point $\phi=0$ after the end of inflation. With each subsequent oscillation, particle creation occurs as $\phi$ crosses zero. This mechanism of particle production is 
described by the theory of preheating in the broad resonance regime [1]. But now we concentrate on the first instant of this process. Remarkably, in certain cases this is all that we need for efficient reheating.

Usually only a small fraction of the energy of the inflaton field $\sim 10^{-2} g^{2}$ is transferred to the particles $\chi$ at that moment (see Eq. (7) in the next section). The role of the parametric resonance was to increase this energy exponentially within several oscillations of the inflaton field. But suppose that the particles $\chi$ interact with fermions $\psi$ with the coupling $h \bar{\psi} \psi \chi$. If this coupling is strong enough, then $\chi$ particles may decay to fermions before the oscillating field $\phi$ returns back to the minimum of the effective potential. If this happens, parametric resonance does not occur. However, as we will show, something equally interesting may occur instead of it: The energy density of the $\chi$ particles at the moment of their decay may become much greater than their energy density at the moment of their creation.

Indeed, prior to their decay the number density of $\chi$ particles, $n_{\chi}$, remains practically constant [1], whereas the effective mass of each $\chi$ particle grows as $m_{\chi}=g \phi$ when the field $\phi$ rolls up from the minimum of the effective potential. Therefore their total energy density grows. One may say that $\chi$ particles are "fattened," being fed by the energy of the rolling field $\phi$. The fattened $\chi$ particles tend to decay to fermions at the moment when they have the greatest mass, i.e. when $\phi$ reaches its maximal value $\sim 10^{-1} M_{p}$, just before it begins rolling back to $\phi=0$.

At that moment $\chi$ particles can decay to two fermions with mass up to $m_{\psi} \sim \frac{g}{2} 10^{-1} M_{p}$, which can be as large as $5 \times 10^{17} \mathrm{GeV}$ for $g \sim 1$. This is two orders of magnitude greater than the masses of the particles which can be produced by the usual mechanism based on parametric resonance [1]. As a result, the total energy density of the produced particles also becomes two orders of magnitude greater than their energy density at the moment of their production. Thus the chain reaction $\phi \rightarrow \chi \rightarrow \psi$ considerably enhances the efficiency of transfer of energy of the inflaton field to matter.

More importantly, superheavy particles $\psi$ (or the products of their decay) may eventually dominate the total energy density of matter even if in the beginning their energy density was relatively small. For example, the energy density of the oscillating inflaton field in the theory with the effective potential $\frac{\lambda}{4} \phi^{4}$ decreases as $a^{-4}$ in an expanding universe with a scale factor $a(t)$. Meanwhile the energy density stored in the nonrelativistic particles $\psi$ (prior to their decay) decreases only as $a^{-3}$. Therefore their energy density rapidly becomes dominant even if originally it was small. A subsequent decay of such particles leads to a complete reheating of the universe.

Since the main part of the process of preheating in this scenario (production of $\chi$ and $\psi$ particles) occurs immediately after the end of inflation, within less than one oscillation of the inflaton field, we will call it instant preheating. We should emphasize that instant preheating is a completely nonperturbative effect, which can lead to the production of particles with momenta and masses many orders of magnitude greater than the inflaton mass. This would be impossible in the context of the elementary theory of reheating developed in [2]. In what follows we will give a more detailed description of the instant preheating scenario.

\section{THE SIMPLEST MODELS}

Consider first the simplest model of chaotic inflation with the effective potential $V(\phi)=\frac{m^{2}}{2} \phi^{2}$, and with the interaction Lagrangian $-\frac{1}{2} g^{2} \phi^{2} \chi^{2}-h \bar{\psi} \psi \chi$. We will take $m=10^{-6} M_{p}$, as required by microwave background anisotropy [10], and in the beginning we will assume for simplicity that $\chi$ particles do not have a bare mass, i.e. $m_{\chi}(\phi)=g|\phi|$. Reheating in this model is efficient only if $g \gtrsim 10^{-4}$ [1], 11 , which implies $g M_{p} \gtrsim 10^{2} m$ for the realistic value of the mass $m \sim 10^{-6} M_{p}$. Thus, immediately after the end of inflation, when $\phi \sim M_{p} / 3$, the effective mass $g|\phi|$ of the field $\chi$ is much greater than $m$. It decreases when the field $\phi$ moves down, but initially this process remains adiabatic, $\left|\dot{m}_{\chi}\right| \ll m_{\chi}^{2}$.

The adiabaticity condition becomes violated and particle production occurs when $\left|\dot{m}_{\chi}\right| \sim g|\dot{\phi}|$ becomes greater than $m_{\chi}^{2}=g^{2} \phi^{2}$. For a harmonic oscillator one has $\left|\dot{\phi}_{0}\right|=m \Phi$, where $\left|\dot{\phi}_{0}\right|$ is the velocity of the field in the minimum of the effective potential, and $\Phi \sim 10^{-1} M_{p}$ is the amplitude of the first oscillation. This implies that the process becomes nonadiabatic for $g \phi^{2} \lesssim m \Phi$, i.e. for $-\phi_{*} \lesssim \phi \lesssim \phi_{*}$, where $\phi_{*} \sim \sqrt{\frac{m \Phi}{g}}$ [1]. Here $\Phi \sim 10^{-1} M_{p}$ is the initial amplitude of the oscillations of the inflaton field. Note that under the condition $g \gg 10^{-4}$ which is necessary for efficient reheating, the interval $-\phi_{*} \lesssim \phi \lesssim \phi_{*}$ is very narrow: $\phi_{*} \ll \Phi$. As a result, the process of particle production occurs nearly instantaneously, within the time

$$
\Delta t_{*} \sim \frac{\phi_{*}}{\left|\dot{\phi}_{0}\right|} \sim(g m \Phi)^{-1 / 2} .
$$

This time interval is much smaller than the age of the universe, so all effects related to the expansion of the universe can be neglected during the process of particle production. The uncertainty principle implies in this case that the created particles will have typical momenta $k \sim$ $\left(\Delta t_{*}\right)^{-1} \sim(g m \Phi)^{1 / 2}$. The occupation number $n_{k}$ of $\chi$ particles with momentum $k$ is equal to zero all the time when it moves toward $\phi=0$. When it reaches $\phi=0$ (or, more exactly, after it moves through the small region $-\phi_{*} \lesssim \phi \lesssim \phi_{*}$ ) the occupation number suddenly (within the time $\Delta t_{*}$ ) acquires the value [1]

$$
n_{k}=\exp \left(-\frac{\pi k^{2}}{g m \Phi}\right)
$$


and this value does not change until the field $\phi$ rolls to the point $\phi=0$ again.

A detailed description of this process including the derivation of Eq. (2) was given in the second paper of Ref. [1]; see in particular Eq. (55) there. This equation (2) can be written in a more general form. First of all, the shape of the effective potential does not play any role in its derivation. The essential point of the derivation of Eq. (2) is that $\chi$ particles are produced in a small vicinity of the point $\phi=0$, when $\phi(t)$ can be represented as $\phi(t) \approx \dot{\phi}_{0}\left(t-t_{0}\right)$. The only thing which one needs to know is not $V(\phi), m$ or $\Phi$, but the velocity of the field $\phi$ at the time when it passes the point $\phi=0$. Therefore one can replace $m \Phi$ by $\left|\dot{\phi}_{0}\right|$ in this equation. Also, the same equation is valid for massive particles $\chi$ as well, if one replaces $k^{2}$ by $k^{2}+m_{\chi}^{2}$, where $m_{\chi}$ is the bare mass of the particles $\chi$ at $\phi=0$. (A similar result is valid for fermions and for vector particles.) Therefore Eq. (2) in a general case (for any $m_{\chi}$ and $V(\phi)$ ) can be written as follows:

$$
n_{k}=\exp \left(-\frac{\pi\left(k^{2}+m_{\chi}^{2}\right)}{g\left|\dot{\phi}_{0}\right|}\right) .
$$

This can be integrated to give the density of $\chi$ particles

$$
n_{\chi}=\frac{1}{2 \pi^{2}} \int_{0}^{\infty} d k k^{2} n_{k}=\frac{\left(g \dot{\phi}_{0}\right)^{3 / 2}}{8 \pi^{3}} \exp \left(-\frac{\pi m_{\chi}^{2}}{g\left|\dot{\phi}_{0}\right|}\right) .
$$

Numerical investigation of inflation in the theory $\frac{m^{2}}{2} \phi^{2}$ with $m=10^{-6} M_{p}$ gives $\left|\dot{\phi}_{0}\right|=10^{-7} M_{p}^{2}$, whereas in the theory $\frac{\lambda}{4} \phi^{4}$ with $\lambda=10^{-13}$ one has a somewhat smaller value $\left|\dot{\phi}_{0}\right|=6 \times 10^{-9} M_{p}^{2}$. This implies, in particular, that if one takes $g \sim 1$, then in the theory $\frac{m^{2}}{2} \phi^{2}$ there is no exponential suppression of production of $\chi$ particles unless their mass is greater than $m_{\chi} \sim 2 \times 10^{15} \mathrm{GeV}$. This agrees with a similar conclusion obtained in [1, 56.

Let us now concentrate on the case $m_{\chi}^{2} \lesssim g\left|\dot{\phi}_{0}\right|$, when the number of produced particles is not exponentially suppressed. In this case

$$
n_{\chi} \approx \frac{\left(g \dot{\phi}_{0}\right)^{3 / 2}}{8 \pi^{3}} .
$$

According to Eq. (3), a typical initial energy (momentum) of each particle $\chi$ at the moment of their production is $\sim(g|\dot{\phi}| \mid / \pi)^{1 / 2}$, so their total energy density is

$$
\rho_{\chi} \sim \frac{\left(g \dot{\phi}_{0}\right)^{2}}{8 \pi^{7 / 2}} .
$$

The ratio of this energy to the total energy density $\rho_{\phi}=$ $\dot{\phi}_{0}^{2} / 2$ of the scalar field $\phi$ at this moment gives

$$
\frac{\rho_{\chi}}{\rho_{\phi}} \sim 5 \times 10^{-3} g^{2} .
$$

This result is practically model-independent, given the interaction term $-\frac{1}{2} g^{2} \phi^{2} \chi^{2}$. In particular, it does not depend on the inflaton mass $m$ in the theory $\frac{m^{2}}{2} \phi^{2}$. The same result can be obtained in the theory $\frac{\lambda}{4} \phi^{4}$ independently of the value of $\lambda$.

An interesting possibility appears if one has $m_{\chi}^{2} \sim$ $g\left|\dot{\phi}_{0}\right|$. Then the probability of production of such particles is not exponentially suppressed during the first oscillation, but it is exponentially suppressed during all subsequent oscillations because $|\dot{\phi}|$ decreases due to the expansion of the universe, and the condition $m_{\chi}^{2} \lesssim g|\dot{\phi}|$ becomes violated. In this case new particles $\chi$ are not created. However, as we already explained, these new particles may not even be necessary. For example, in the theory $\frac{\lambda}{4} \phi^{4}$ the energy density of the inflaton field $\rho_{\phi}$ decreases as $a^{-4}$, whereas the energy density stored in the nonrelativistic particles $\chi$ (prior to their decay) decreases only as $a^{-3}$. Therefore their energy density rapidly becomes dominant even if originally it was small. Their subsequent decay makes the process of reheating complete.

But preheating in our model becomes much more efficient if we use the mechanism described in the beginning of this paper. Indeed, let us assume that the particles $\chi$ survive until the field $\phi$ rolls up from $\phi=0$ to the point $\phi_{1}$ from which it returns back to $\phi=0$. In the theory $\frac{m^{2}}{2} \phi^{2}$ one has $\phi_{1} \approx 0.07 M_{p}$, whereas in the theory $\frac{\lambda}{4} \phi^{4}$ one has $\phi_{1} \approx 0.12 M_{p}$. We will take $\phi_{1} \approx 0.07 M_{p}$ in our estimates. At that time the mass of each particle $\chi$ will be $g \phi_{1} \sim 10^{-1} g M_{p}$, they will be nonrelativistic, and their total energy density (for the case of the theory $\left.\frac{m^{2}}{2} \phi^{2}\right)$ will be

$$
\rho_{\chi}=m_{\chi} n_{\chi} \approx 10^{-1} g M_{p} \frac{\left(g \dot{\phi}_{0}\right)^{3 / 2}}{8 \pi^{3}} \sim 10^{-14} g^{5 / 2} M_{p}^{4} .
$$

Therefore the ratio of the energy density of $\chi$ particles to the energy density of the inflaton field $\sim \dot{\phi}_{0}^{2} / 2$ will be

$$
\frac{\rho_{\chi}}{\rho_{\phi}} \sim 10^{-3}\left|\dot{\phi}_{0}\right|^{-1 / 2} M_{p} g^{5 / 2} \sim 2 g^{5 / 2} .
$$

The last result follows from the relation $\left|\dot{\phi}_{0}\right| \sim$ $10^{-1} m M_{p} \sim 10^{-7} M_{p}^{2}$ for $m \sim 10^{-6} M_{p}$. Under the condition $g \gtrsim 10^{-4}$, which is the standard condition for efficient preheating [1], this ratio is much greater than the one in Eq. (7).

If the particles $\chi$ do not decay when the field $\phi$ reaches $\phi_{1}$, then their energy will decrease again in parallel with $|\phi|$, until it reaches the value given by Eq. (7). Thus, preheating is most efficient if all particles $\chi$ can decay at the moment when the field $\phi$ reaches its maximal value $\phi_{1}$. This is possible if the lifetime of the particles $\chi$ created at the moment $t_{0}$ is close to $\Delta t \sim \pi m^{-1} / 4$. Particles $\chi$ in our model can decay to fermions, with the decay rate [1]

$$
\Gamma(\chi \rightarrow \psi \psi)=\frac{h^{2} m_{\chi}}{8 \pi}=\frac{h^{2} g|\phi|}{8 \pi} .
$$


Note that the decay rate grows with the growth of the field $|\phi|$, so particles tend to decay at large $|\phi|$. One can easily check that the particles $\chi$ decay when the field $\phi$ reaches its maximal value $|\phi| \approx 0.07 M_{p}$ if

$$
h^{2} g \sim \frac{500 m}{M_{p}} \sim 5 \times 10^{-4} .
$$

At the moment when $|\phi|$ reaches $0.07 M_{p}$, the particles $\chi$ have effective mass $m_{\chi}=g|\phi| \sim 0.07 g M_{p}$. Such particles can decay to two fermions $\psi$ if $m_{\psi}<0.035 \mathrm{gM}_{p}$. This implies that after the first half of an oscillation, the scalar field $\phi$ can produce fermions with mass up to $0.035 \mathrm{~g} M_{p}$. For example, in the theory with $g \sim 10^{-1}, h \sim 7 \times 10^{-2}$ one can produce fermions with mass up to $m_{\psi} \sim 4 \times 10^{16}$ $\mathrm{GeV}$, and in the theory with $g \sim 1, h \sim 2 \times 10^{-2}$ one can produce particles with mass up to $4 \times 10^{17} \mathrm{GeV}$.

As we have found, initially the ratio $\frac{\rho_{\chi}}{\rho_{\phi}}$ is suppressed by the factor $2 g^{5 / 2}$, see Eq. (9). But this suppression is not very strong, and if the energy density of the $\psi$ particles during some short period of the evolution of the universe decreases not as fast as the energy density of the inflaton field and other products of its subsequent decay, then very soon the universe will be dominated by the products of decay of the particles $\psi$, and reheating will be complete.

If $h^{2} g \gg 5 \times 10^{-4}$, the $\chi$ particles may decay before the oscillating field $\phi$ reaches its maximal value $\phi_{1} \sim 10^{-1} M_{p}$. This can make our mechanism somewhat less efficient. However, the decay cannot occur until $m_{\chi}=g|\phi|$ becomes greater than $2 m_{\psi}$. If, for example, the fermions have mass $\sim 0.03 g M_{p}$, then the decay occurs only when the field $\phi$ reaches its maximal value $\phi_{1}$ even if $h^{2} g \gg 5 \times 10^{-4}$. This preserves the efficiency of our mechanism even for very large $h^{2} g$.

On the other hand, for $h^{2} g \ll 5 \times 10^{-4}$, the particles $\chi$ do not decay within a single oscillation. In this case the parametric resonance regime becomes possible, which again leads to efficient preheating according to [1]. Moreover, superheavy fermions still will be produced in this regime, because the oscillating field will spend a certain amount of time at $\phi \sim \phi_{1}$. During this time superheavy particles will be produced, and their number may not be strongly suppressed.

The mechanism of particle production described above can work in a broad class of theories. For example, one can consider models with the interaction $\frac{g^{2}}{2} \chi^{2}(\phi+v)^{2}$. Such interaction terms appear, for example, in supersymmetric models with superpotentials of the type $W=$ $g \chi^{2}(\phi+v)$ [12]. In such models the mass $m_{\chi}$ vanishes not at $\phi_{1}=0$, but at $\phi_{1}=-v$, where $v$ can take any value. Correspondingly, the production of $\chi$ particles occurs not at $\phi=0$ but at $\phi=-v$. When the inflaton field reaches the minimum of its effective potential at $\phi=0$, one has $m_{\chi} \sim g v$, which may be very large. If one takes $v \sim M_{p}$, one can get $m_{\chi} \sim g M_{p}$, which may be as great as $10^{18} \mathrm{GeV}$ for $g \sim 10^{-1}$, or even $10^{19} \mathrm{GeV}$ for $g \sim 1$.
If, however, one takes $v \gg M_{p}$, the density of $\chi$ particles produced by this mechanism will be exponentially suppressed by the subsequent stage of inflation. This possibility will be discussed in the next section.

Since parametric amplification of particle production is not important in the context of the instant preheating scenario, it will work equally well if the inflaton field couples not to bosons but to fermions 13,14. Indeed, the creation of fermions with mass $g|\phi|$ also occurs because of the nonadiabaticity of the change of their mass at $\phi=0$. The theory of this effect at $g \gtrsim 10^{-4}$ is very similar to the theory of the creation of $\chi$ particles described above; see in this respect [14]. The efficiency of preheating will be enhanced if the fermions $\psi$ with a growing mass $g|\phi|$ can decay into other fermions and bosons, as in the scenario described in the previous section.

It is amazing that oscillations of the field $\phi$ with mass $m=10^{13} \mathrm{GeV}$ can lead to the copious production of superheavy particles with masses 4 - 5 orders of magnitude greater than $m$. The previously known mechanism of preheating was barely capable of producing particles of mass $\sim 10^{15} \mathrm{GeV}$, which is somewhat below the GUT scale, and even that was possible only in the strong coupling limit $g=O(1)$. Our new mechanism allows for the production of particles with mass greater than $10^{16}$ $\mathrm{GeV}$ even if the coupling constants are relatively small. This fact may play an important role in the theory of baryogenesis in GUTs.

\section{FAT WIMPZILLAS}

Until now we have discussed a new mechanism of preheating. However, recently there has been a growing interest in the possibility of the production of superheavy WIMPS after inflation 15 17,7]. Such particles (which have been proudly called WIMPZILLAS [18]) could be responsible for the dark matter content of the universe, and, if they have very large but finite decay time, they can also be responsible for cosmic rays with energies greater than the Greisen-Zatsepin-Kuzmin limit [19]. The focus of these works in a certain sense was opposite to that of the theory of preheating: It was necessary to find a mechanism for the production of stable (or nearly stable) particles which would survive until now. For that purpose, the mechanism of their production must be extremely inefficient, since otherwise the present density of such relics would be unacceptably large.

As one could expect, it is much easier to make the mechanism inefficient rather than the other way around. For example, our Eq. (4) implies that the probability of production of superheavy $\chi$ particles is suppressed by a factor of $\exp \left(-\frac{\pi m_{\chi}^{2}}{g\left|\dot{\phi}_{0}\right|}\right)$. In the theory $\frac{m^{2}}{2} \phi^{2}$ with $m=10^{-6} M_{p}$ we have $\left|\dot{\phi}_{0}\right|=10^{-7} M_{p}^{2}$, so this suppression factor is given by $\exp \left(-\frac{10^{7} \pi m_{x}^{2}}{g M_{p}^{2}}\right)$. This implies that for $g \sim 1$ the production of particles with $m_{\chi} \sim 10^{16} \mathrm{GeV}$ 
is suppressed approximately by $10^{-10}$, and this suppression becomes as strong as $10^{-40}$ for $m_{\chi}=2 \times 10^{16} \mathrm{GeV}$. This same level of suppression can be achieved, for example, with $g=10^{-2}$ and $m_{\chi}=2 \times 10^{15}$. Thus, by fine-tuning of the parameters $m_{\chi}$ and $g$ one can obtain any value of the density of WIMPS at the present stage of the evolution of the universe. This result agrees with the result obtained in [7] by a different method.

This suppression mechanism is equally operative for the process $\phi \rightarrow \chi \rightarrow \psi$ discussed in our paper. If the particles $\chi$ are heavy at $\phi=0$, their number will be exponentially suppressed. When the field $\phi$ grows, their masses grow as follows: $m_{\chi}^{2}(\phi)=m_{\chi}^{2}+g^{2} \phi^{2}$, At the moment of their decay these particles can have mass of the order $10^{17}-10^{18} \mathrm{GeV}$. The main advantage of this new mechanism is that the process of fattening of the particles $\chi$ described above allows for the production of particles $\psi$ which can be $10^{2}$ times heavier than their cousins discussed in 15 17, [7]. In the absence of established terminology, one can call such superheavy particles FAT WIMPZILLAS.

Another way to produce an exponentially small number of superheavy WIMPS is to produce them at the last stages of inflation. This is possible in theories with the interaction term $\frac{g^{2}}{2} \chi^{2}(\phi+v)^{2}$, as described in the previous section. If one takes $v \gtrsim M_{p}$, then the particles $\chi$ will be created during inflation. The number of $\chi$ particles produced during inflation in the simplest theory with $V(\phi)=\frac{m^{2}}{2} \phi^{2}$ does not depend on $v$ because $\dot{\phi}$ does not depend on $\phi$ and on $v$ in this scenario: $\dot{\phi}=\frac{m M_{p}}{2 \pi}$ [10]. However, their density will subsequently be exponentially suppressed by inflation. This is exactly what we need if the $\chi$ particles or the products of their decay are WIMPS. For example, in the theory with $V(\phi)=\frac{m^{2}}{2} \phi^{2}$ the universe inflates by a factor of $\exp \left(2 \pi v^{2} / M_{p}^{2}\right)$ after the creation of $\chi$ particles [10], so their density at the end of inflation becomes smaller by a factor of $\exp \left(6 \pi v^{2} / M_{p}^{2}\right)$. This leads to a desirable suppression for $v \sim 2 M_{p}$. (The exact number depends on the subsequent thermal history of the universe.) Meanwhile the masses of WIMPS produced by this mechanism can be extremely large, of order $g M_{p}$. If the $\chi$ particles are stable, they themselves may serve as superheavy WIMPS with nearly Planckian mass. If they decay to fermions, then the fermions may play a similar role.

\section{QUINTESSENCE, INSTANT PREHEATING, AND BLACK HOLE PRODUCTION}

The mechanism of instant preheating works even better in models with potentials of a "quintessential" type. For example, one may consider potentials $V(\phi)$ which behave (approximately) as $\frac{m^{2}}{2} \phi^{2}$ or $\frac{\lambda}{4} \phi^{4}$ at $\phi<0$, and (gradually) vanish when $\phi$ becomes positive. Models of this type were first proposed by Ford and Spokoiny, and recently they have been revived by several other authors [8]. These models for a long time were unpopular because the field $\phi$ in these models does not oscillate and therefore the old mechanism of reheating based on the perturbative decay of the inflaton field [2] in such models cannot work. The only known way to reheat the universe in this scenario was to invoke the gravitational creation of particles due to the expansion of the universe [20,8]. This mechanism of reheating is relatively inefficient and may lead to several cosmological problems which we will discuss in a separate publication [21]. Fortunately, our mechanism works in these theories as well. Indeed, the theory of production of particles $\chi$ in our scenario is applicable to theories with any $V(\phi)$; the number of produced particles depends only on $g$ and $\left|\dot{\phi}_{0}\right|$. In the theories where $V(\phi)$ gradually vanishes at large $\phi$, the field $\phi$ rolls at least up to $\phi \sim M_{p}$ before it stops (or turns back). Then the particles $\chi$ acquire masses $m_{\chi} \sim g M_{p}$. If the coupling constant $g$ is sufficiently large $\left(g \sim 10^{-1}\right)$, the decay of $\chi$ particles may produce superheavy particles $\psi$ with masses up to $10^{17}-10^{18} \mathrm{GeV}$. Since the value of the field $\phi$ at that time is approximately one order of magnitude greater than in the usual model with the potential $\frac{m^{2}}{2} \phi^{2}$, whereas the energy density $\rho_{\phi}$ will be decrease due to the expansion of the universe, the energy density of particles $\chi$ at that time will be much greater than $2 g^{5 / 2} \rho_{\phi}$.

It is instructive to compare the density of particles produced by this mechanism to the density of particles created during gravitational particle production, which is given by $\rho_{\chi} \lesssim O\left(H^{4}\right) \sim \rho_{\phi} \frac{\rho_{\phi}}{M_{p}^{4}}$. In the simplest models of chaotic inflation $\frac{\rho_{\chi}}{\rho_{\phi}} \sim \frac{\rho_{\phi}}{M_{p}^{4}} \sim 10^{-14}$ at the end of inflation, and in hybrid inflation models it is even much smaller. Thus, for $g \gtrsim 10^{-6}$ the number of particles produced during instant preheating is much greater than the number of particles produced by gravitational effects. Therefore one may argue that reheating of the universe in theories with quintessential potentials should be described using the instant preheating scenario. As for the gravitational particle production studied in [8], it still can be useful, perhaps not as a mechanism of reheating, but as a source of WIMPS. Indeed, as we already mentioned, the number of WIMPS must be extremely small, so the relative inefficiency of the gravitational particle production is quite appropriate in this context [16].

In theories with quintessential potentials the energy density of the inflaton field $\phi$ rolling along the flat direction of the effective potential decreases as $a^{-6}$, i.e. much faster than the energy density of other particles. Thus, in such theories instant preheating is amazingly efficient. Even if the particles $\chi$ decay to relativistic particles immediately after they are produced, so that the fraction of energy transferred to particles from the inflaton field initially is only $5 \times 10^{-3} g^{2}$, still their energy density will dominate as soon as the size of the universe grows by a factor of $15 g^{-1}$. Meanwhile, if one uses our favorite mechanism by which the $\chi$ particles "fatten" before de- 
caying, then the energy density of produced particles becomes much greater, and the period of their dominance with respect to the energy density of the classical inflaton field begins even much earlier, when the scale factor grows by only a factor of $O\left(g^{-5 / 6}\right)$.

Note that in this scenario we still assume that $\chi$ particles eventually decay to some other particles. Otherwise the backreaction of the created $\chi$ particles can stop the growth of the field $\phi$ and return it back to $\phi=0$. However, since the number of produced particles and their interaction with the field $\phi$ are suppressed for small $g$, the backreaction of the created particles becomes significant only after a time interval much greater than $m^{-1}$ [21. As a result, this scenario will work under much milder constraints on $g$ and $h$ than the scenarios in which $\phi$ oscillates about a minimum of $V(\phi)$.

One can avoid this problem altogether if $V(\phi)$ becomes flat not at $\phi=0$, but only at $\phi \gtrsim M_{p}$. In such a case the backreaction of created particles never turns the scalar field $\phi$ back to $\phi=0$. Therefore the decay of the particles $\chi$ may occur very late, and one can have efficient preheating for any values of the coupling constants $g$ and $h$.

One may also consider a scenario in which the particles $\chi$ are stable, and have bare mass $m_{\chi} \sim 10^{16} \mathrm{GeV}$. The probability of production of such particles will be exponentially suppressed, but then their masses will increase by $g|\phi|$, which can be very large. This allows to produce superheavy WIMPS of mass $m_{\chi} \gg 10^{16} \mathrm{GeV}$ without assuming that $\chi$ particles must decay to $\psi$ (see also the previous section).

Finally, let us assume that the $\chi$ particles are stable, and have bare mass $m_{\chi} \ll 10^{16} \mathrm{GeV}$. Then the probability of creation of such particles will be large, and if they cannot decay to other particles, we will eventually end up with the universe filled by an unacceptably large number of superheavy WIMPS. However, if the field $\phi$ continues rolling for a very long time, it may reach values much greater than $M_{p}$. In such a scenario, the particle masses $g|\phi|$ at some moment may become even larger than $M_{p}$.

The possibility of producing superheavy particles with masses exceeding $M_{p}$ should be addressed in the framework of superstring theory. It is rather interesting that superstring theory in certain cases may be important for the description of reheating after inflation, which was viewed as a low-energy phenomenon.

In conventional quantum field theory, an elementary particle of mass $M$ has a Compton wavelength $M^{-1}$ smaller than its Schwarzschild radius $2 M / M_{p}^{2}$ if $M>$ $M_{p}$. Therefore one may expect that as soon as $m_{\chi}=g|\phi|$ becomes greater than $M_{p}$, each $\chi$ particle becomes a Planck-size black hole, which immediately evaporates and reheats the universe. This is a very unusual (and admittedly very speculative) version of the instant preheating scenario which deserves more detailed investigation.

\section{CONCLUSIONS}

The theory of reheating after inflation is already rather old. For many years we thought that the classical oscillating inflaton field could be represented as a collection of scalar particles of mass $m \lesssim 10^{13} \mathrm{GeV}$, that each particle decayed to particles of smaller mass, and that our final goal was to calculate the reheating temperature $T_{r}$.

During the last few years we have learned that this simple picture in certain cases can be very useful, but typically one must use the nonperturbative theory of reheating for the description of the first stages of reheating. The main ingredient of this theory was the theory of broad parametric resonance. Particle production in this scenario could be represented as a series of successive acts of creation, during which the number of produced particles increased exponentially. It seems now that this was only a first step towards a complete understanding of nonperturbative mechanisms of reheating after inflation. As we have shown in this paper, preheating may occur in a different way. It may be sufficient to consider a single act of creation, especially if one takes into account the relative increase of energy of produced particles during the subsequent evolution of the classical inflaton field, and the possibility of the chain reaction $\phi \rightarrow \chi \rightarrow \psi$. This new mechanism is capable of producing particles of nearly Planckian energy, which was impossible in the previous versions of the theory of reheating.

One of the key ingredients of the nonperturbative mechanism of preheating described above is a nonadiabatic change of the mass $m_{\chi}(\phi)$ near the point where it vanishes (or at least strongly decreases). Such situations occur very naturally in supersymmetric theories of elementary particles if one identifies $\phi$ and $\chi$ with moduli fields which correspond to flat directions of the effective potential. Indeed, in supersymmetric theories the effective potential often has several flat directions, which may intersect. When one of the moduli fields (the inflaton) moves along a flat direction and reaches the intersection, the mass of another field vanishes. A simplest example of this situation was described in Sect. III. The change of the number of massless degrees of freedom is a generic phenomenon which is under intense investigation in the context of supersymmetric gauge theories, supergravity and string theory, where it is associated with the points of enhanced gauge symmetry, see e.g. 22,23.

Masses of elementary particles may also change nonadiabatically during cosmological phase transitions. At the moment of a phase transition masses of some particles vanish and may even temporarily become tachyonic. In this case particle production may become even more intense.

Our main conclusion is that with an account taken of the new possibilities discussed above the scenario of preheating becomes more robust. In the cases where parametric resonance may occur, it provides a very efficient mechanism of preheating. Now we have found that effi- 
cient preheating is possible even in models where parametric resonance does not happen because of the rapid decay of produced particles. Instant preheating occurs in the usual inflationary models where the inflaton field oscillates near the minimum of its effective potential. But this mechanism works especially well in models with effective potentials which slowly decrease at large $\phi$, as in the theory of quintessence. The conversion of the energy of the inflaton field to the energy of elementary particles in these models occurs very rapidly, and it is always $100 \%$ efficient. A preliminary investigation indicates that in some versions of such models preheating may even produce particles of mass greater than $M_{p}$ which become black holes and immediately evaporate. It would be very interesting to investigate this possibility in the context of string theory.

\section{ACKNOWLEDGMENTS}

It is a pleasure to thank R. Kallosh, S. Shenker, A. Starobinsky, S. Thomas, and A. Vilenkin for useful discussions. This work was supported by NSF grant AST9529-225. The work of A.L. was also supported by NSF grant PHY-9870115.

[1] L.A. Kofman, A.D. Linde, and A. A. Starobinsky, Phys. Rev. Lett. 73, 3195 (1994); L.A. Kofman, A.D. Linde, and A. A. Starobinsky, Phys. Rev. D 56, 3258 (1997); P.B. Greene, L.A. Kofman, A.D. Linde, and A. A. Starobinsky, Phys. Rev. D 56, 6175 (1997).

[2] A.D. Dolgov and A.D. Linde, Phys. Lett. 116B, 329 (1982); L.F. Abbott, E. Fahri, and M. Wise, Phys. Lett. 117B, 29 (1982); A.A. Starobinsky, in: Quantum Gravity, Proc. of the Second Seminar "Quantum Theory of Gravity" (Moscow, 13-15 Oct. 1981), eds. M.A. Markov and P.C. West (Plenum, New York, 1984), p. 103.

[3] A.D. Linde, Phys. Lett. 108B, 389 (1982); A. Albrecht, P.J. Steinhardt, M.S. Turner and F. Wilczek, Phys.Rev.Lett. 48, 1437 (1982).

[4] L.A. Kofman, A.D. Linde, and A. A. Starobinsky, Phys. Rev. Lett. 76, 1011 (1996); I. I. Tkachev, Phys. Lett. B376, 35 (1996); S. Khlebnikov, L. Kofman, A.D. Linde, and I. Tkachev, Phys. Rev. Lett. 81, 2012 (1998), hepph/9804425; I. Tkachev, S. Khlebnikov, L.A. Kofman, and A.D. Linde, hep-ph/9805209.

[5] E.W. Kolb, A.D. Linde, and A. Riotto, Phys. Rev. Lett. 77, 4290 (1996); E.W. Kolb, A. Riotto, and I.I. Tkachev, Phys. Lett. B423, 348 (1998).

[6] S.Yu. Khlebnikov and I.I. Tkachev, Phys. Lett. B390, 80 (1997); B.R. Greene, T. Prokopec and T.G. Roos, Phys. Rev. D 56, 6484 (1997); I. Zlatev, G. Huey, P.J. Steinhardt, Phys. Rev. D 572152 (1998).
[7] D.J.H. Chung, hep-ph/9809489.

[8] L.H. Ford, Phys. Rev. D 35, 2955 (1987); B. Spokoiny, Phys. Lett. B 315, 40 (1993); M. Joyce, Phys. Rev. D 55, 1875 (1997); M. Joyce and T. Prokopec, Phys. Rev. D 57, 6022 (1998); P.J.E. Peebles and A. Vilenkin, astro$\mathrm{ph} / 9810509$.

[9] R.R. Caldwell, R. Dave, and P.J. Steinhardt, Phys. Rev. Lett. 80, 1582 (1998).

[10] A.D. Linde, Particle Physics and Inflationary Cosmology (Harwood, Chur, Switzerland, 1990).

[11] Note that if one takes $g>10^{-3}$, radiative corrections to the effective potential may considerably change its shape [10]. However, this does not happen in supersymmetric theories where the contributions of fermions and bosons nearly cancel each other.

[12] A. Berera and T.W. Kephart, hep-ph/9811295.

[13] J. Baacke, K. Heitmann, and C. Patzold, hepph/9806205.

[14] P.B. Greene and L.A. Kofman, hep-ph/9807339.

[15] V. Berezinsky, M. Kachelriess, and A. Vilenkin, Phys. Rev. Lett. 79, 4302 (1997); V.A. Kuzmin and V.A. Rubakov, Phys. Atom. Nucl. 61, 1028 (1998); Yad. Fiz. 61, 1122 (1998), astro-ph/9709187.

[16] D.J.H. Chung, E.W. Kolb, and A. Riotto, hepph/9802238; V.A. Kuzmin and I.I. Tkachev, hepph/9802304, hep-ph/9809547.

[17] D.J.H. Chung, E.W. Kolb, and A. Riotto, hep$\mathrm{ph} / 9809453$.

18] E.W. Kolb, D.J.H. Chung, and A. Riotto, hep$\mathrm{ph} / 9810361$.

[19] K. Greisen, Phys. Rev. Lett. 16, 748 (1966); G.T. Zatsepin and V.A. Kuzmin, Pisma Zh. Eksp. Teor. Fiz. 4, 114 (1966).

[20] Ya. B. Zel'dovich and A. A. Starobinsky, Zh. Exp. Theor. Fiz. 61, 2161 (1971) [Sov. Phys. JETP 34, 1159 (1972)]; L.P. Grishchuk, Lett. Nuovo Cim. 1260 (1975), Sov. Phys. JETP 40, 409 (1975); S. G. Mamaev, V. M. Mostepanenko, and A. A. Starobinsky, ZhETF 70, 1577 (1976) [ Sov. Phys. JETP 43, 823 (1976)]; A.A Grib, S.G. Mamaev, V.M. Mostepanenko, Gen. Rel. Grav. 7535 (1976); A.A. Grib, S.G. Mamaev, and V. M. Mostepanenko, Vacuum Quantum Effects in Strong Fields (Energoatonizdat, Moscow, 1988).

[21] G. Felder, L.A. Kofman, A.D. Linde, and A.A. Starobinsky, in preparation.

[22] C.M. Hull and P.K. Townsend, Nucl. Phys. B451, 525 (1995); E. Witten, Nucl. Phys. B443, 85 (1995); K. Intriligator and N. Seiberg, Nucl. Phys. Proc. Suppl. 45BC, 1 (1996); M. Shifman, Prog. Part. Nucl. Phys. 39, 1 (1997).

[23] J. Polchinski, String Theory (Cambridge Univ. Press, Cambridge 1998). 